\title{
Unveröffentlichte Liszt-Briefe aus Weimar und Dresden
}

\author{
Klára HAMBURGER \\ Ungarische Akademie der Wissenschaften \\ Deres u. 12, H-1124 Budapest, Ungarn \\ E-Mail: hambivan@t-online.hu
}

(Received: April 2014; accepted: September 2014)

\begin{abstract}
This documentary contains 16 Liszt-letters preserved at the Goethe-SchillerArchiv (GSA) in Weimar and further 14 items from the Sächsische Landesbibliothek - Staats- und Universitätsbibliothek (SLUB) in Dresden. The Weimar letters include those in which Liszt addressed (in French) Ignaz Moscheles and Julius Benedict, both German musicians living in London, about his 1840 concert tour in England. Also, he wrote in French to singer Pauline Viardot-Garcia, Madame Érard, and his Neapolitan pupil Luisa Cognetti. His letters in German to Hermann Levi deal with Richard Wagner. In another letter Liszt is asking the Vienna Home Secretary Baron Alexander von Bach, to have his Gran Mass published at the state administration's expense. His letters to Count Sándor Teleki and Ede Reményi concern Hungarian musical life. Liszt is giving instructions for the publishing of his work Hymne de l'enfant à son réveil to his Hungarian publisher Nándor Táborszky and writing a dry refusal to his former Hungarian pupil Sándor Bertha. The envelope of a letter to Madame Munkácsy has a mistake in the orthography of the family name. The documents from Dresden include an Albumblatt Liszt wrote for Clara Schumann, a recommendation for Heinrich Ehrlich, the composer of the first Lento-theme of Liszt's Hungarian Rhapsody no 2. Further letters were written to Laura Kahrer (one of them having been published in a slightly altered manner by La Mara) and a series of eight letters to Liszt's Swiss disciple Bertrand Roth.
\end{abstract}

Keywords: Liszt, correspondence 
In meiner letzten größeren Veröffentlichung von Franz Liszts Briefen habe ich mit Bedauern feststellen müssen:

Leider ist die Unveröffentlichkeit der Briefe Liszts seit dem Tode des hervorragenden Lisztianers Charles Suttoni nicht mehr mit absoluter Bestimmtheit nachzuweisen: Es hat sich noch niemand gefunden, der seine unentbehrliche Publikation Liszt Correspondence in Print: An Expanded, Annotated Bibliography (= Journal of the American Liszt Society 25 [January-June 1989]) und Liszt Correspondence in Print: A Supplemental Bibliography (= ibid. 46 [Fall 1999]) fortgesetzt hätte. ${ }^{1}$

Diese Einsicht hat sich bei der Vorbereitung jener geplanten Publikation von Briefen Liszts aus dem Bestand des Goethe-Schiller-Archivs, deren Ergebnis ich hiermit vorlege, erneut bewahrheitet. Die zur Publikation vorgesehenen Briefe, die ich mit Hilfe von Frau Dr. Evelyn Liepsch, Leiterin der Musikabteilung des GSA, bestellt und erhalten habe, verteilte ich in drei Gruppen:

a) 16 deutsche und französische Briefe, an verschiedene Adressaten, unter ihnen mehrere Ungarn;

b) 16 französische Briefe an Jessie Laussot;

c) 9 französische Briefe an das Ehepaar Zarębski.

Bald stellte es sich heraus, dass Malou Haine in ihrem Artikel „Dix-neuf lettres de la correspondance entre Liszt et les époux Zarembski“ außer den Weimarer Briefen aus Archiven von Antwerpen und New York auch noch Weitere publizierte. ${ }^{2}$ Auch mein zweites Projekt musste ich aufgeben, als ich erfuhr, dass Maria-Teresa Storino die Veröffentlichung der Briefe Liszts an Laussot vorbereitete. $^{3}$

Es blieben mir also von allen, aus Weimar übersandten Briefen nur die hier folgenden sechzehn zur Veröffentlichung übrig. Sie erscheinen in der Originalsprache, d. h. deutsch bzw. französisch, in chronologischer Reihenfolge.

Ignaz Moscheles und Julius Benedict, die Adressaten der ersten zwei Briefe, waren beide deutschsprachige, in London lebende Künstler, denen Liszt jedoch auf Französisch schrieb, da er zur Zeit die deutsche Sprache nicht gut beherrschte.

1. Klára Hamburger, „Unbekannte Liszt-Dokumente aus deutschen Bibliotheken“, Studia Musicologica 53/4 (September 2012), 383-457, hier 384.

2. Malou Haine, „Dix-neuf lettres de la correspondance entre Franz Liszt et les époux Zarembski“, Liszt Saeculum 58 (1997/1), 13-26.

3. Ihre Ausgabe ist inzwischen auch erschienen: „New Liszt Letters to Jessie Laussot“, in Franz Liszt, Lectures et écritures, hrsg. von Florence Fix, Laurence Le Diagon-Jacquin und Georges Zaragoza (Paris: Herrmann, 2013), 131-148. 
Seine Konzerttournee in England (1840) wie auch die Herausgabe seiner Werke in London versuchte er mit Hilfe dieser Musiker vorzubereiten.

Im französischen Brief an Dr. Froriep (Nr. 3) geht es um die Goethe-Stiftung.

Drei französische Briefe (Nr. 5, 10, 11) sind an verschiedene Damen geschrieben. Einer an die bewunderte Freundin und ehemalige Schülerin Liszts, die Sängerin Pauline Viardot-Garcì; einer an ein Mitglied der befreundeten Pariser Klavierfabrikanten-Familie, Madame Érard, und einer an seine Schülerin in Rom, die gebürtige Neapolitanerin Luisa Cognetti.

Zwei der Briefe (auf Deutsch) stehen in Zusammenhang mit Richard Wagner: Einen schrieb Liszt an den Dirigenten Hermann Levi (Nr. 12), den anderen (Nr. 15) an einen unbekannten deutschen Musikprofessor. Auf Deutsch schrieb Liszt auch an den Klavierfabrikanten Bechstein (Nr. 14), im Interesse des von ihm hochgeschätzten Kirchenmusikers Pater Joseph Mohr. Im Anhang veröffentliche ich einen französischen Liszt-Brief an Mohr.

Die weiteren Briefe stehen alle in Zusammenhang mit Ungarn. Es war bekannt, dass die Graner Messe auf Staatskosten gedruckt und herausgegeben wurde. Nun liegt das Dokument vor, dass Liszt den berüchtigten österreichischen Innenminister, Baron Alexander von Bach (im deutschen Brief Nr. 4), selber darum gebeten hatte. Ein Zeugnis echter Männerfreundschaft und Vaterlandsliebe ist der französische Brief an den Grafen Sándor Teleki und ein Teil von dessen Antwort (Nr. 6). Freundschaftliche Ratschläge (auf Französisch) an den getreuen Freund, den Violinisten Ede Reményi, finden sich in Brief Nr. 8. Liszts Sinn für Selbstironie bezeugen seine Dankworte (auf Französisch) an das Witzblatt Borsszem Jankó [Jankó Pfefferkorn] (Nr. 7) für die (beigelegten) Karikaturen. Seinem ungarischen Verleger Nándor Táborszky gibt Liszt detaillierte Anweisungen (auf Deutsch, Nr. 9) bezüglich der Veröffentlichung seines Hymne de l'enfant à son réveil. Seinem einstigen Verehrer und Schüler, dem in Paris lebenden Komponisten und Kritiker Sándor Bertha, der zum Feind der „Zukunftsmusik“ geworden ist, (Nr. 13), schreibt der sonst immer so höfliche Meister (auf Französisch) sehr schroff und zurückweisend. An Madame Cécile, die französische Gattin des ungarischen Malers Mihály Munkácsy, adressiert, ist in Weimar bloß ein leeres Couvert zu finden, das einen Schreibfehler im Namen der Adressatin aufweist (Munkascy statt Munkácsy, Nr. 16).

Das Datum der Briefe habe ich überall vereinheitlicht und vor die Briefe gestellt. Die Orthographie entspricht überall genau jener der Autographe, ausgenommen die bei Liszt mit einer Linie über den Buchstaben markierten Verdoppelungen.

In der Sächsischen Landesbibliothek - Staats- und Universitätsbibliothek Dresden (SLUB) gibt es mehr als fünfzig autographe Liszt-Dokumente. Der größte Teil (mehrheitlich an den Dichter, Journalist und Theaterintendant Franz von Dingelstedt geschrieben) wurde längst von La Mara [Marie Lipsius] in ihrer Ausgabe von Franz Liszts Briefen veröffentlicht. Dennoch ist es mir gelungen, einige 
noch unbekannte Schriftstücke mit der Hilfe freundlicher Mitarbeiterinnen der SLUB zu finden, ${ }^{4}$ die hier publiziert werden sollen.

Das erste Dokument (Nr. 17) ist ein musikalisches Albumblatt für Klavier von drei Takten in A-Dur, geschrieben für Clara Schumann, datiert 27 May [18]40, Unterschrift: F. Liszt.

Zwei Briefe sind an Unbekannte geschrieben: der eine (Nr. 18, auf Französisch, datiert Weimar, 4. Februar 1850) an eine Gräfin, der andere (Nr. 19, datiert Weimar, 29. April 1884) an einen Herren, interessanterweise in deutscher Schrift, deren Liszt sich seit seiner Jugend nicht mehr bediente. Beide haben etwas Gemeinsames: Sie geben uns eine Vorstellung von Liszt, dem Organisator, der mit großen Mengen von dilettantischen Musikstücken überschüttet wurde, und diese auf eine unübertrefflich höfliche Weise zurückzuweisen verstand. Der zweite französische Brief (Nr. 22, datiert Weimar, 28. August [ohne Jahreszahl, aber bestimmt nach 1851]) ist ein Empfehlungsschreiben für den in Wien geborenen Pianisten, Komponisten und Musikschriftsteller Heinrich Ehrlich, der u. a. auch in Ungarn konzertierte (1844: Klausenburg [heute: Cluj, Rumänien]; 1845: Pest). Ehrlich behauptete, die Melodien, die dem ersten Lento-Thema in Liszts Ungarischer Rhapsodie Nr. 2 folgen, seien seine Kompositionen. In seinem Buch Dreissig Jahre Künstlerleben teilte er auch Liszts französischen Brief (datiert Rom, 30. 03. 1860) mit, in welchem der ungarische Meister diesen Tatbestand anerkannte. ${ }^{5}$ Der Adressat des Empfehlungsschreibens, Herr Lindemann aus Hannover, war ein ausgezeichneter Amateur-Cellist, der an einem von Liszt 1851 in Bad Eilsen für die Harfenistin Rosalie Spohr organisierten Konzert mitwirkte.

Die weiteren Liszt-Briefe in Dresden stammen alle aus dem Nachlass von Künstlern, die am dortigen Konservatorium unterrichteten bzw. selber bei Liszt studiert hatten.

Die in Mistelbach bei Wien geborene Laura Kahrer (nach ihrer Heirat mit dem Violinisten Eduard Rappoldi Rappoldi-Kahrer genannt) war eine der herausragendsten Pianistinnen ihrer Zeit. Sie wurde von Joseph Dachs (Wien), Liszt (Weimar, 1870, 1873), Adolf Henselt (Sankt Petersburg) und Hans von Bülow (Bad Salzungen, Liebenstein) ausgebildet. 1890 wurde sie Klavierpädagogin am Dresdner Konservatorium, 1911 ebenda zur Professorin ernannt. Sie hinterließ eine lange Reihe von Kompositionen und einige musikalische Schriften. ${ }^{6}$

In Dresden sind zwei an Laura Kahrer gerichtete Briefe Liszts mit Couvert aufbewahrt (Nr. 20 und 21). Der erste Brief (Wien, 15. April 1869) wurde ohne Couvert von La Mara auf ihre Art, etwas ,verbessert“ herausgegeben; es schadet daher nicht, ihn nochmals getreu, samt Couvert zu veröffentlichen. Der Zweite

4. Meinen besonderen Dank möchte ich Frau Kerstin Schellbach von der Auskunft SLUB Dresden aussprechen, die so freundlich war, mir die Signaturen und die Adressaten der Briefe einzeln anzugeben.

5. Heinrich Ehrlich, Dreissig Jahre Künstlerleben (Berlin: Steinitz,1893), 116-118.

6. Silke Wenzel, „Laura Rappoldi-Kahrer“, in Musik und Gender im Internet (MUGI), Musikerinnenlexikon, hrsg. von Beatrix Borchard - Nina Noeske. mugi.hfmt-hamburg.de (Stand: 04. 10. 2014). 
jedoch (Pest, 13. April 1874) ist meines Wissens bisher unbekannt geblieben. Aus diesem wird uns klar, wie sehr Liszt den ungestörten Urlaub Hans von Bülow in Deutschland versichern wollte, aber auch, dass er Laura Kahrer dennoch mit diesem Empfehlungsbrief zu ihm schickte (und mit Erfolg!).

Die Briefe Nr. 23-30 stammen aus dem Nachlass von Liszts schweizerischem Schüler, dem Pianisten und Komponisten Bertrand Roth (1855, Degersheim, Kanton St. Gallen - 1938, Bern). 1858 übersiedelte die Familie Roth nach Pirna, wo Vater J. J. Fr. Roth die erste deutsche Stickereifabrik einrichtete. Bertrand studierte Philosophie, dann wurde er Musikschüler im Leipziger Konservatorium. 1877 begann er sein Studium bei Franz Liszt, den er auf zwei Reisen nach Pest und Rom begleitete. Bereits 1879 trat er als Solist beim Allgemeinen Deutschen Musikfest auf und konzertierte in verschiedenen Städten Deutschlands, Italiens und der Schweiz. Als Klavierlehrer arbeitete er im Hoch'schen Konservatorium in Frankfurt am Main, wurde dort einer der Gründer, dann Mitdirektor des Raff'schen Konservatoriums. In Dresden wurde er 1884 zum Königlichen Musikprofessor ernannt. In seinem Musiksalon fanden zwischen 1901 und 1930 dreihundert Sonntagsmatineen mit Werken von zeitgenössischen Komponisten statt. ${ }^{7}$

Von den an Bertrand Roth (bzw. an seinen Vater J. J. Fr. Roth) geschriebenen Briefen liegt ein Inventar vor, betitelt „Nachlass Bertrand Roth“ mit Unterschrift und Stempel: „Mit meinen besten Grüssen Jörg Bertrand Müller [Stempel: Dr. Jörg Müller-Schmidt, Brunnadernstrs. 92, 3006 Bern], im August 2000“. Der Enkel des Komponisten erklärt darin, die der SLUB geschickten Liszt-Autographe seien sein Eigentum, und fügt hinzu:

\footnotetext{
Im Nachlass von Prof. Bertrand Roth befand sich ein von ihm selbst beschrifteter Briefumschlag:

Briefe und Karten von Franz Liszt - Joachim Raff - Hofrat Pudor. ${ }^{8}$

Dieser Briefumschlag steckte in einem etwas grösseren Briefumschlag, mit folgender handschriftlicher Inhaltsangabe aus der Feder von Albertine Roth [Tochter Bertrands]:

8 Lisztbriefe [sic] an Bertrand Roth (einer davon an den Vater J. J. Fr. Roth). Alle 8 Briefe sind vorhanden, in ihren ursprünglichen Umschlägen. 2 Umschläge haben Visitenkarten-Format, mit Visitenkarten von Franz Liszt. 5 Briefe sind durch die Post befördert. Die Briefe sind von meiner Mutter mit I-VIII bezeichnet, in chronologischer Reihenfolge.
}

Ich hielt mich streng an die Orthographie und den Inhalt der Autographe, ausgenommen das Datum, welches in einheitlicher Form den Briefen vorangestellt wurde. 


\section{1. \\ GSA 59/71,11 \\ An Ignaz Moscheles (London) \\ [sechs beschriebene Seiten, der Schluss fehlt]}

Milan, 28 Decembre 1837

Je suis extrêmement reconnaissant, mon cher Monsieur Moscheles, de toutes les peines que vous voulez bien prendre à l'occasion de mes manuscrits. En m'adressant directement à vous pour cette affaire à laquelle, soit à tort, soit à raison, j'attache assez d'importance, je savais d'avance que la question se simplifierait à raison de votre loyauté et de votre amicale complésance. La dernière lettre que vous me faites l'amitié de m'ecrire, me prouve que je ne m'étais point trompé ; recevez donc de nouveau mes plus affectueux remerciements.

Je crois ne point en abuser sur ma position d'artiste en général. Je sais que les 4 ou 5 années qui se sont écoulées entre ma carrière d'enfance et le commencement de ma periode virile (de 1829 à 33 ou 34) m'ont été facheux sous plusieurs rapports. Les maladies presque constants qui m'eprouvaient alors, et le profond et amer decouragement qui a fait la visite, sans parler ici d'autres causes qu'y s'y joignirent, ont retardées sans doute de beaucoup le déploiement extérieur de mes facultés. // Quoique depuis l'age de 18 ans je me sois toujours assez serieusement occupé de composition, je n'ai pourtant pu qu'en publier qu'un petit nombre d'ouvrages et dans ce petit nombre même il s'en trouve peutêtre une moitié qui n'a absolument aucune recommandation auprès des MMrs les Editeurs de Musique. - Vous voyez mon cher Monsieur Moscheles, que je ne me fais nullement illusions, et que je vais au devant les objections. - Toutes fois, le hazard ayant voulu qu' à l'aide de quelques articles de journaux, les uns elogieux, les autres presqu'enfiévrileux, mon nom penetre dans quelques parties de l'Allemagne et d'Italie, voire même en France, où je suis en quelque sorte fixé et où par conséquant j'ai eu à soutenir les plus ennuyeuses comparaisons, les plus sottes querelles, pour confirmer l'ancien Proverbe « Nul n'est prophète dans son propre pays ![ »] :- le même // hazard a encore voulu que MMrs Hoffmeister ${ }^{9}$ à Leipzig, Mr Haslinger ${ }^{10}$ à Vienne, $\mathrm{Mr}$ Ricordi $^{11}$ à Milan, et plusieurs Editeurs de Paris me faisaient des offres sinon très brillantes, du moins acceptables pour mes compositions à venir. MMrs Hoffmeister et Ricordi par exemple se sont engagés à prendre la totalité de mes oeuvres à raison d'un Louis par 4 pages (6 frcs par pages). S'il m'est permis de vous le dire,

\footnotetext{
9. Friedrich Hofmeister, deutscher Musikverleger.

10. Tobias Haslinger, Musikverleger und Komponist in Wien. Er und sein Sohn Carl waren gute Freunde Liszts.

11. Der berühmte, bis heute funktionierende Musikverlag wurde von Giovanni Ricordi gegründet. Sein Nachfolger wurde sein Sohn Tito.
} 
j'espère et ferai en sorte que ces Messieurs n'aient jamais à se repentir de leurs avances. - C'est un arrangement de cette nature que j'avais proposé à Mr Mori ${ }^{12}$ avec lequel j'ai eu autrefois des relations. Je ne tiens nullement que ce soit lui qui devienne l'Editeur de mes oeuvres à Londres ; entre nous soit dit, je préfererai même que ce fit la maison Adisson et Beale. ${ }^{13}$ Mais je lui avais ecrit pour vous epargner // l'ennui de discussion d'Editeur.

Je regrette beaucoup que ma lettre à Mr Mori ne vous ait pas été remise d'abord ; cela eut abrégé les affaires. C'est par erreur que Mr Pupp ${ }^{14}$ la lui a envoyé directement.

En resumé voici le contenu de cette lettre.

Je lui proposai d'editer environ 220 pages (au plus 260) par an, à raison d'une Guinée (minimum) les 4 pages. Dans son intérêt et dans le mien je le priai de donner à ces ouvrages la plus grande publicité possible. Les ouvrages que je pourrai immédiatement livrer sont les suivans

1. Collections des Symphonies de Beethoven (Partitions de Piano. $)^{15}$

2. Grandes Etudes ${ }^{16}$

3. Grand Galop Chromatique (en forme de Caprice) ${ }^{17} / /$

4. Grand morceau de Concert, composé par MMrs Thalberg, Herz, Czerny, Chopin. Introduction, Stretta et Finale par F. Liszt. (Pour ce morceau seulement comme le produit en est destiné à mon oeuvre de charité et qu'il ne m'appartient que par comission, je lui demandai je crois le même prix que m'en donne Haslinger à Vienne c'est à dire 18 à 20 Guinées.

5. Impressions et Poesies, Suite et complément de l'Album d'un Voyageur Ire Année. ${ }^{18}$

(Tout ces ouvrages seront edités en même temps en Allemagne, en France et en Italie.)

Si donc vous pensez que MMrs Addison et Beale vient disposé à prendre avec moi (grace à votre intervention) un arrangement pour un an, dixhuit mois, jusqu’à mon voyage d'Angleterre enfin, à raison d'une Guinée par 4 pages, je vous au-

12. Nicholas Mori, englischer Musikverleger und Violinvirtuose italienischer Abstammung. Liszts Verhandlungen mit den englischen Verlagen endeten ergebnislos.

13. Thomas Beale, Musikverleger in London, der den Verlag zusammen mit Robert Addison, später mit dem deutschen Pianisten Johann Baptist Cramer leitete. Drei Briefe Liszts an Heinrich Schlesinger, die Beale erwähnen, wurden von Michael Short veröffentlicht als „Letters 28-30“ in Liszt Letters in the Library of Congress (Hillsday, New York: Pendragon, 2003), 25-27.

14. Mr Pupp konnte nicht identifiziert werden.

15. LW, S. 468, R. 168

16. LW, S. 137, R. 2a

17. LW, S. 219, R. 41

18. LW, S. 156 , R. 8 
torise pleinement à m'engager vis à vis d'eux. Il est bien entendu // que si vous jugez à propos de demander davantage, et si vous êtes assez heureux pour obtenir davantage, je ne vous désavouerai point. Pour moins, il me serait probablement difficile de l'accepter.

Pardon, cent fois pardon encore mon cher Monsieur Moscheles, de cette longue et trop_longue lettre; mais à plus de deux cents lieus de distance il comporte d'être à la fois précis et détaillé.

Je vous remercie cordialement des bonnes propositions que vous me faites pour ma venue en Angleterre. ${ }^{19}$ Vous serez assurément la première personne que j'irai trouver en arrivant à Londres. Malheureusement, à cause de plusieurs travaux commencés et que j'ai à coeur de poursuivre activement, j'ajournerai encore d'un an ma tour d'Outre mer.

L'année prochaine, je la passerai toute entière en Italie. ${ }^{20} \mathrm{Si}$ je pouvais par hazard vous y être de quelque utilité //

[Der Schluß fehlt.]

\section{2. \\ GSA 59/57, 33 \\ An Julius Benedict (London) ${ }^{21}$ \\ [vier Seiten]}

Vienne, 30 Janvier [1840]

Cent fois merci Mon cher Benedict, de votre amicale invitation. Je m'y rendrai exactement, quoiqu'il me faille pour cela faire plus de 300 lieues ! - J'espère que vous ne serez point mecontant de moi et que les Anglais me trouveront much improuved.

Je me rejouis beaucoup de ce voyage, et si j’ai tant tardé // à le faire j'espère que personne n'avoir perdu à attendre. À l'heure qu'il est ; me voici arrivé de pieds en cap - quand nous nous sommes vus la dernière fois à Paris, il s'en fallait de beaucoup. Je vous suis grand gré du bon souvenir que vous avez bien voulu garder de moi,

Comptez toujours sur mon // plus affectueux devouement, et croyez que je serai heureux de vous le temoigner de mon mieux.

Tout à vous

F. Liszt

19. Schließlich machte Liszt vom Mai bis Ende Juni 1840 die England-Tournee.

20. Liszt hielt sich vom 17. August 1837 bis November 1839 in Italien auf, unterbrochen mit seinen Wiener Konzertserien.

21. Julius Benedict, deutscher Komponist und Dirigent. Er war tätig im Kärntnertortheater in Wien und im Teatro San Carlo, Neapel. Seit 1835 lebte er in London. 
Soyez assez bon pour me rappeler très affectueux au souvenir de Moscheles, pour lequel, vous le savez, j'ai toujours professé estime particulière. Haslinger m'a communiqué son Concerto pastorale. ${ }^{22}$ C'est une oeuvre trés distinguée comme toutes celles qu'il a ecrites // en dernier lieu.

\section{3. \\ GSA 06/4658a \\ An Dr. Robert Froriep ${ }^{23}$ \\ [zwei Seiten]}

[Mit Couvert, mit Stempel, wahrscheinlich eingehändigt:]

\section{Monsieur Monsieur Frohriep / FLiszt}

[Weimar], 5 Mai 1851

Je vous suis sensiblement obligé, Monsieur, de la lettre que vous avez bien voulu m'ecrire, et sur le contenu de laquelle les circonstances donneront, je le présume plus de joies par la suite. En vous en faisant aussitôt mes sincéres remerciemens, j'ajouterai simplement qu'un Institut, tel que devra être la Fondation-Goethe ${ }^{24}$ ne peut se réaliser qu'avec le concours sincère et unanime de toutes les forces actives, intelligentes et noblement intentionnés de Weymar. Or comme par vos antécédents et vos aboutissans // vous prenez evidemment rang et place dans cette catégorie trop restreinte hélas! Je me plais à croire que les quelques differences de point de vue, qui nous séparent encore s'effaceront aisément sur le terrain de la pratique, qui en cet ordre de matières, est le terrain viril par excellence.

Veuillez bien agréer, Monsieur je vous prie, l'expression des sentimens les plus distinguées

et les plus affectueux

de votre tout dévoué

FLiszt

22. Ignaz Moscheles: Klavierkonzert Nr. 8, op. 96.

23. Dr. Robert Froriep, Arzt, Geheimrat in Berlin. Ab 1846 leitete er in Weimar einen Verlag für geographische und ärztliche Bücher.

24. Die Goethe-Stiftung lag Liszt sehr am Herzen. In seinem Essay „De la Fondation Goethe à Weimar“ entfaltete er seine wichtigen künstlerischen Ansichten. Siehe Franz Liszt, „Zur Goethe Stiftung“ (1850), in ders., Gesammelte Schriften, Bd. V: Streifzüge - Kritische, polemische und zeithistorische Essays, hrsg. von Lina Ramann (Leipzig: Breitkopf und Härtel, 1882), 1-109. 


\section{4. \\ GSA 59/05 \\ An Baron Alexander von Bach ${ }^{25}$ \\ [Konzept, zwei Seiten und ein zweites Konzept von Seite 1]}

Wien, [?] Sept. [18]56

Eure Excellenz,

Die Fürsorge und Protection welche Eure Excellenz den geistigen Interessen im Kaiserstaate angedeihen lassen gestatten mir den Wunsch und die Bitte vorzutragen, die Messe welche ich im Auftrage Seiner Eminenz den Fürst Primas von Ungarn $^{26}$ zur Einweihungs Feierlichkeit der Graner Basilica componirt und daselbst am 31ten August zur Aufführung gebracht habe, durch die K.K. Staatsdruckerei, Partitur und Clavierauszug, auf Staatskosten gedrukt und herausgegeben werden.

Ohne mein Werk ungeziemend // anzupreisen, erlaube ich mir bescheidentlich die Zuversicht auszusprechen dass der catholische Sinn und Geist welcher demselben zu Grunde liegt und dessen verschiedenen Bestandteile erfüllen durch die Veröffentlichung allmählich sicher Verbreitung und Verständniss finden werde so dass ich hoffen dürfte einen nicht unwürdigen Beitrag zur Christlichen Kunst so wie der hohen Kirchen und Landes Feier des 31 August geliefert zu haben,

In der Erwartung dass meine Bitte jenem fördernden Wohlwollen begegnet dessen ernste und bedeutende Kunstbefehlungen bedürftig sind habe ich die Ehre gehorsamst zu verbleiben

Eurer Excellenz

unterthänigster und ergebenster

Diener

FLiszt

25. Baron Alexander von Bach, österreichischer Jurist, 1849-1859 Innenminister des Kaiserstaates. Nach der Unterdrückung des ungarischen Freiheitskampfes 1848-1849 wurde in Ungarn ein nach ihm benanntes, zentralisiertes, auf Spitzelei beruhendes Repressaliensystem eingeführt. Von 1859 bis 1867 diente dann Bach als österreichischer Gesandter beim Heiligen Stuhl in Rom. Da er die Graner Messe auf Staatskosten drucken ließ, wurde er von Liszt hochgeschätzt: Der Meister hatte selbst vor, ihn aufzufordern, sein Trauzeuge zu sein. Siehe Liszts Brief in: Franz Liszt. Briefe aus ungarischen Sammlungen 1835-1886, hrsg. von Margit Prahács (Budapest - Kassel: Akadémiai Kiadó - Bärenreiter, 1966), 115.

26. János Scitovszky, Fürstprimas von Ungarn. 


\title{
5. \\ GSA 59/76,26 \\ An Pauline Viardot ${ }^{27}$ \\ [eine beschriebene Seite]
}

[Weimar, um April-Juni 1870]

Très chère Admirabilissime,

Certainement et triplement le mot en voyelles : Oui je viendrai vous prendre ce soir [eingefügt: ( 8 heures)] pour vous accompagner chez les Milde. ${ }^{28}$ Mademoiselle Bertha Lewin ${ }^{29}$ est invité chez Mme de Meyendorff ${ }^{30}$ demain, - et le reste suivra, de mieux en mieux.

Bien à vous de

vieille amitié

[Unterschrift fehlt]

\author{
6. \\ An Sándor Graf Teleki ${ }^{31}$ \\ [Konzept, eine Seite mit vielen Korrekturen \\ + die letzte Seite des Briefes von Teleki]
}

[ohne Ort und Datum, wahrscheinlich Pressburg, Ende 1872]

Très cher incomparable [extraordinaire],

Ton Salut en t'honoriaste, et les pages qui suivent m'ont profondément ému et emerveillé.

C'est la glorification a perpétuelle de notre amitié si noblement engagée de ta part par un duel à Berlin en 1842, et noblement maintenu pendant 40 années. L'élévation de sentiments et pensées s'exprime dans un style flamboyant, digne de Victor Hugo. L'extraordinaire inclut le vrai. Oui, très cher ami, simplement

27. Der junge Liszt gab Pauline Viardot García als kleinem Mädchen in Paris Klavierstunden. Sie wurden bis an Liszts Lebensende gute Freunde. Der Meister schrieb auch ein Essay über sie. Siehe Franz Liszt, „Pauline Viardot-Garcia“ (1859), in ders., Gesammelte Schriften, hrsg. von Lina Ramann, Bd. III (Leipzig: Breitkopf und Härtel, 1881), 121-135.

28. Hans Feodor von Milde, Bariton, und seine Frau, die Sopranistin Rosa, geb. Aghte, Sänger in Weimar.

29. Sängerin, Schülerin von Pauline Viardot.

30. Olga von Meyendorff, geb. Füstin Gortschakowa, intime Freundin Liszts.

31. Sándor Graf Teleki, einer der besten ungarischen Freunde Liszts. 1842 hatte er für ihn in Berlin duelliert, begleitete ihn auf seine Konzerttournee in Ost-Europa und Russland. Am 13. März 1879 schrieb Liszt an die Fürstin Wittgenstein aus Klausenburg: „Mein alter, intimer Freund, der Graf Sándor Teleki, schrieb einen fulguranten Artikel über mich.“ Siehe Franz Liszt's Briefe, Bd. 7, Briefe an die Fürstin Carolyine Sayns-Wittgenstein, 4, herausgegeben von La Mara [Marie Lipsius] (Leipzig: Breitkopf und Härtel, 1902), 244. 
dit « Patrie et honneur » reste notre devise, et nous s'en tenierons à la pratique de [notre] mieux à la Vérité et à l'honneur de notre patrie et de nous mêmes.

À revoir à Budapest, où je retournerai droit d'ici, mi Janvier et resterai jusqu'aux premiers jours d'avril, où nous arrangerons [quand] nous serons chez toi, à Kolto, ${ }^{32}$ à davantage de ton fidèle, tout devoué vieux ami

\section{[Letzte Seite der Antwort Telekis:]}

un artiste, et ses créations sont la gloire de notre patrie, même si claire que tu as éveillé n'appartenant pas tous à notre nation, mais c'est ici que tu leur a communiqué le feu

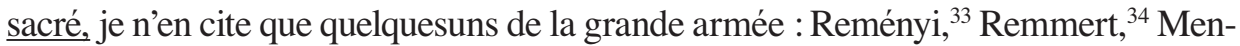
ter, ${ }^{35}$ Joseffi ${ }^{36}$ et Zichy. ${ }^{37}$ Cet homme n'a qu'une main, et il joue comme avec six, il est artiste en tout, il pense et il écrit, il sent vivement et profondément, il est compositeur.

Et maintenant permets moi de te tendre la main, reçois la poignée de main de la cordiale hospitalité, ce n'est pas seulement moi, mais la ville entière qui se réjouit de ta présence.

$$
\underline{\text { Salut!!! }}
$$

Sándor Teleki

\section{7. \\ GSA 59/76,22 \\ An das Witzblatt Borsszem Jankó \\ [zwei beschriebene Seiten +8 Bilder $]^{38}$}

[Budapest, zwischen dem 30. Oktober und Ende des Jahres 1873], Mardi soir

Mille remerciements, chers Messieurs ; et à revenir chez vous, demain soir

affectueusement

F. Liszt //

Post Scriptum

Après les « $\mathrm{Ta}$-ta $-\mathrm{ta} »$ viendront les $« \mathrm{Ti}-\mathrm{ti}-\mathrm{ti}$, les To - to - to, les Tou - tou tou »-- et je n'ai plus nul courage à faire le « piou - piou - piou»

32. Zu diesem Besuch in Koltó kam es erst 1885. Siehe ibid., 414-416.

33. Ede Reményi (Hoffmann), ungarischer Violinist, war einer der treuesten Anhänger und Freunde Liszts.

34. Martha Remmert, deutsche Pianistin, Schülerin Liszts.

35. Sophie Menter, deutsche Pianistin, Schülerin Liszts.

36. Rafael Joseffy, ungarischer Pianist, Schüler Liszts.

37. Géza Graf Zichy, einarmiger Pianist, späterer Freund Liszts.

38. Beigelegt acht Karikaturen von Liszt, welche in der Zeitschift erschienen: Borsszem Jankó 6/275 (6. April 1873), 3-5. Siehe http://epa.oszk.hu/01300/01338/00275/pdf/00275.pdf 


\section{8. \\ GSA 59/72,2 \\ An Ede Reményi \\ [eine beschriebene Seite]}

Weimar, 17 Juin 1873

Cher ami,

«Multa Paucis $»^{39}$ - Donc vos Concerts d'été, ad libitum, selon l'opportunité, [seront] nécessaires à votre rentrée [diese zwei Worte gestrichen] au théatre comme " Concertmeister $»{ }^{40}$ Elle dépend d'Erkel, ${ }^{41}$ Richter, ${ }^{42}$ - et surtout des sourdines que l'ami Reményi saura mettre à son violon. Choisissez les avec discernement et circonspection.

À revoir, fin d'Octobre à Budapest.

Très affectueux hommages à Mme Remenyi ${ }^{43}$ et cordialement à vous

F. Liszt

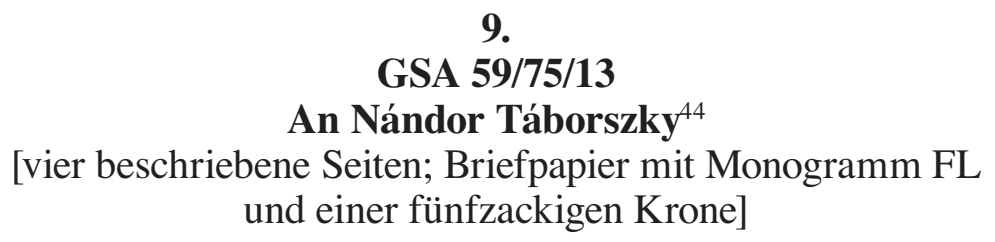

Villa d'Este, 14 August [18]74

Geehrter Freund,

Einige Tage später als Ihr Brief erhielt ich das Manuscript der Kindlichen Hym$n e .{ }^{45}$ Die deutsche Übertragung von Cornelius ${ }^{46}$ ist vortrefflich - ganz nach Erwartung und Wunsch gelungen. Daneben erfreute mich die Calligraphie unseres Freundes Gobbi. ${ }^{47}$ Sagen Sie Ihm meinen besten Dank dafür.

39. Viel sagen in wenigen Worten, ,kurzum“.

40. Ede Reményi war 1869-1870 Konzertmeister im Orchester des Ungarischen Nationaltheaters. Anscheinend unternahm er 1873 einen Versuch, die Stelle wieder zu bekommen, der jedoch scheiterte.

41. Ferenc Erkel war seit 1838 erster Kapellmeister des Nationaltheaters.

42. Hans Richter war ab 1871 Dirigent, dann 1874-1875 Operndirektor im Nationaltheater.

43. Reményi hatte 1872 Gizella Fáy, die Tochter des Komponisten und Pianisten Antal Fáy, geheiratet.

44. Nándor Táborszky war einer der Eigentümer des 1868 gegründeten ungarischen Musikverlages Táborszky \& Parsch. Von Anfang der siebziger Jahre bis zu Liszts Tod war ihr Verhältnis freundschaftlich.

45. Hymne de l'enfant à son réveil. Text von Alphonse de Lamartine, LW, S. 19, R. 508, dritte Fassung. Liszts.

46. Peter Cornelius, deutscher Komponist und Dichter, gehörte zum engsten Weimarer Freundeskreis

47. Henrik Gobbi, ungarischer Komponist, Dirigent und Pianist, Freund Liszts. 
Habet Entschuldigung des Wegstreichens (mit Bleistift) der hemmenden $\underline{9}$ und 4 Bezeichnungen. Zuviel Taktschlägerei verleidet bereits die Musik ${ }^{48}$ anstatt solche Unwirtschaft aufzumuntern soll man trachten sie abzuschaffen und möglichst // auf ein feineres Tact Gefühl hinweisen.

Nächstens schicke ich Ihnen das Manuscript zurück. Nun ist es ein Tripel Autograph geworden, mittelst Gobbi, Cornelius und meiner Wenigkeit.

Eingedenk des unbewährten Axioms der alten Physik: „Natura abhorret a vacuo“" (Natur verabscheuet die Leere) benützte ich die zufällig leer gelassene Zeile in der Abschrift Gobbis um einen Harfen Part, den ich früher schreiben wollte, zu fertigen. Derselbe wird Sie in keine übermässige Kosten stürzen, da er sich nur auf einer einzigen Zeile bewegt.

Also die Auflage der Hymne derartig:

A)Die kleine 6 zeilige Partitur in klein 8tav Format, mit französisch, // deutsch und ungarischem Text,

B)Die 3 Singstimmen, Sopran und Alt, gleichfalls mit dreifachem Text versehen.

C)Die 2 begleitenden Instrumente, Harmonium und Harfe separat obendrein, mit einer oberen Zeile der principal Singstimme. - wo der erste Sopran pausirt der 2te - durchgängig vom ersten bis zum letzten Takte, gestochen, und mit Text en français, deutsch und magyar beschwert... Wofür ich Sie bitte, den Stecher besonders aufmerksam zu machen.

In dem Tripel Autograph steht der Titel deutsch und französisch, nebst Anmerkung betreffs der Aufführung, und die Dedication des Opus an den Pester „Liszt Verein“. ${ }^{49}$ Für die ungarische Übersetzung zu sorgen, überlasse ich meinem // verständigen, im Persönlichen hartnäckigsten, insbesonders lieben Freunde Ábrányi,$^{50}$ der mir innigst verwandt bleibt im Magyarismus. ${ }^{51}$

Senden Sie mir hieher die Correcturen der Hymne nebst die Manuscripte und bewahren Sie dann dieses in ihrer Privatsammlung, als ein Zeichen, - wertlos auf dem öffentlichen Markte - meiner persönlichen Zuneigung.

Freundschaftlich ergebenst

F. Liszt

48. Siehe das Essay Liszts „Ein Brief über das Dirigieren. Eine Abwehr“ (1853), in ders., Gesammelte Schriften, hrsg. von Lina Ramann, Bd. V (Leipzig: Breitkopf und Härtel, 1881), 227-232.

49. Der Pester Liszt-Verein wurde von Kirchenkapellmeister Mátyás Engeszer und seiner Frau, der Sängerin Katalin Marsch, gegründet. Frau Engeszer besuchte Liszt am 13. Dezember 1870 mit einigen Damen, und bat ihn um Erlaubnis für den Frauenchor, seinen Namen tragen zu dürfen. Später wurde das Ensemble zum gemischten Chor ergänzt.

50. Kornél Ábrányi d. Ä., ungarischer Komponist und Musikschriftsteller.

51. Ein Ausdruck Liszts für seine Vaterlandsliebe. 
Die 2 ersten Bände der Moskauer Edition von Chopin, sind mir richtig hinzugekommen, und ich danke Ihnen für die Sendung der Bücher, welche Baron Augusz $^{52}$ freundlichst bestellte.

Correcturen des Franciscus de Paule ${ }^{53}$

erwartet

FL

[Oben, quer über der Seite:]

Bis Ende Januar verbleibe ich hier in ruhig arbeitender Abgeschiedenheit. Danach - zur Fasten Conzert Saison komme ich zurück - Fischplatz. ${ }^{54}$ Budapest.

10.

GSA 59/76,37

An Madame Érard

[zwei Seiten]

Budapest, 6 Décembre, [18]74.

Chère Madame,

Vraiment, la Maison Erard était pour moi une maison paternelle, dès mon arrivée à Paris, en 1823. Vos très honorés Oncles, Jean Baptiste et Sebastien, prirent en affection le petit Liszt, qui tant bien que mal eut l'honneur de produire premièrement en public, à Paris et à Londres, l'excellente invention de Sebastien Erard, du piano à double échappement. //

Que de souvenirs me rattachent aux Erards ! ... Mon père et ma mère leur etaient reconnaissants ; et ma fille, Cosima, partage mon sentiment de fidélité.

Comment vous dire, chère Madame, mon regret de ne pouvoir remplir le souhait que Mr Reményi m'exprime de votre part ? Veuillez m'excuser de n'être plus qu'un ex-pianiste invalide, - et agréez mes sincères hommages, de vieille affection

F. Liszt

\footnotetext{
52. Baron Antal Augusz, der beste ungarische Freund Liszts, in dessen Haus in Szekszárd er öfters zu Gast war.

53. LW, S. 175, R. 17/2 Legende Nr. 2, E-Dur: Der Hl. Franciscus auf den Wogen schreitend.

54. Am Fischplatz (Hal tér) in der Nähe der Donau hatte Liszt seine erste Wohnung in Pest. Beim Bau der ersten Elisabethbrücke um 1900 wurde der Platz eingeebnet.
} 


\section{1. \\ GSA 59/62,3 \\ An Luisa Cognetti ${ }^{55}$ \\ [eine beschriebene Seite]}

[Oben, quer:] Mademoiselle Louisa Cognetti, 74 - Via Monte di Dio, Napoli

Mademoiselle,

Villa d'Este, 20 Octobre [18]77

Maintes fois de très agréables nouvelles de vos succès artistiques me parviennent, et dernièrement encore $\mathrm{Mr}$ Sgambati ${ }^{56}$ me parlait de votre talent, avec de sincères éloges.

Je m'y associe bien volontiers en souvenir de votre aimable visite à la Villa d'Este ; et vous prie, Mademoiselle, de compter sur mes meilleurs sentiments distingués et affectueux

F. Liszt

\section{2. \\ GSA 59/70,12 \\ An Hermann Levi ${ }^{57}$}

[Drei beschriebene Seiten. Seiten 1-2 und die obere Hälfte von Seite 3 beschrieben in deutscher Schrift von fremder Hand, mit Liszts deutschen Verbesserungen in lateinischer Schrift. Seite 2 und die obere Hälfte von Seite 3 sind gestrichen. Untere Hälfte von Seite 3 von der Hand Liszts mit lateinischen Buchstaben. Die autographen Teile von mir hervorgehoben - K. H.]

Weimar, 23ten Juli [18]83

Hochverehrter Herr,

Die Fortsetzung der Bayreuther Wagner Festspiele hängt in letzter Linie und in letzter Instanz von der Gnade Seiner Majestät des Königs von Bayern ${ }^{58}$ ab. Bis zu dessen Allerhöchsten Entscheidung werden jetzt nur vorbereitende Schritte zu thun seyn, die Betheiligung meiner Person aber unwichtig erscheint.

Mein vierzigjähriger verehrter Freund Dr. Gille ${ }^{59}$ aber // wird der Conferenz in Bayreuth, nächsten Freitag beiwohnen, und meine Ansicht über die Sachlage verdeutlichen.

55. Luisa Cognetti, italienische Pianistin, seit 1876 Schülerin Liszts in Rom.

56. Giuseppe Sgambati, italienischer Komponist, Dirigent und Pianist. Liszts Schüler in Rom.

57. Hermann Levi, deutscher Dirigent, dem Richard Wagner die Leitung der Uraufführung des Parsifal am 26. Juli 1882 anvertraute.

58. Ludwig II., König von Bayern.

59. Dr. Carl Gille, der in Jena lebende Jurist, gehörte zu Liszts intimsten Freunden. 
Für die ehrenvolle Auszeichnung welche mir die in Bayreuth versammelten Kunstgenossen gewähren, sagt verbindlichsten Dank60

hochachtungsvoll ergebenst

FL

Beiliegend der mir mitgetheilte Circular Entwurf

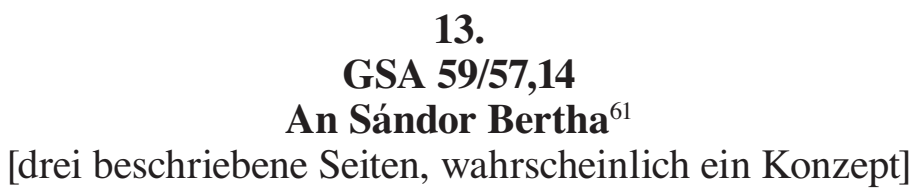

Weimar, 21. Octobre [18]84

Cher Compatriote,

Passons sur votre « retour aux idées conservatrices » en Musique. Mes amis et moi, nous prétendons non sans raison d'être et de rester fort conservateurs en $\mathrm{Pa}$ lestrina, Lassus, Bach, Händel, Haydn, Mozart, Beethoven. ++ // [Eingeschobene Zeilen auf Seite 3:] ++ les chef d'oeuvres de ces grands maîtres sont au mieux dirigés, interprétés, commentés, édités par des artistes non hostiles aux tendences actuelles. Je citerai seulement trois noms en Allemagne: F. Witt, ${ }^{62} \mathrm{Ch}$. Riedel, ${ }^{63}$ H. de Bulow. Peu à peu on finira par s'entendre et de ne pas confondre la stagnation avec la conservation. ++ //

Au sujet du Concert de bienfaisance austro-hongrois veuillez avoir l'obligeance de dire au président du Comité que je dois rester en dehors du programme. Lors du Concert pour le monument de Beethoven à Vienne, ${ }^{64}$ j’ai déclaré publiquement que désormais mes vieux doigts cesseraient leur office trop prolongé en public.

Personne ne saurait exiger que s’inflige un démenti quelconque

F. Liszt

60. Wahrscheinlich meint Liszt damit, dass er zum Präsidenten der Festspiele 1884 erwählt wurde.

61. Sándor (Alexandre de) Bertha, Komponist, Pianist und Musikschriftsteller. Als Schüler von Mihály Mosonyi wurde er zum Anhänger der Musik von Liszt und Wagner. Von 1862 an lebte er in Leipzig, 18641865, der Einladung Liszts folgend, in Rom. Danach ließ er sich in Paris nieder, änderte seine musikalischen Ansichten radikal und wurde zum Neo-Konservativen.

62. Franz Xaver Witt, deutscher Kirchenmusiker, einer der Leiter der Cäcilianer-Bewegung.

63 Carl Riedel, Chorleiter in Leipzig, einer der Gründer des Allgemeinen Deutschen Musikvereins, dessen Präsident er nach Franz Brendels Tod (1868) wurde.

64. Das Beethoven-Denkmal in Wien von Caspar von Zumbusch (der Bildhauer hatte auch Liszts Büste angefertigt) wurde am 1. Mai 1880 enthüllt. Liszt spielte Beethovens Klavierkonzert Es-Dur in einem Konzert zu Gunsten des Denkmals am 18. März 1877 in Wien. Sein Gelübde, nicht mehr öffentlich Klavier zu spielen, hatte er übrigens noch mehrfach gebrochen. 


\section{4. \\ GSA 69/57/9 \\ An Friedrich Wilhelm Carl Bechstein ${ }^{65}$ \\ [zwei beschriebene Seiten]}

München, 19ten October [18]85

Sehr geehrter Herr und Freund,

Der sehr geist- und verdienstreiche Professor J. Mohr ${ }^{66}$ wünscht ihrer Freundlichkeit empfohlen zu sein. Seine catholischen Kirchen Gesangs Bücher sind sehr verbreitet (in französischen und deutschen Ausgaben) und zu schätzen. Er schreibt Ihnen angelegentlich der Bezahlung eines // Pianinos welches Ihnen hoffentlich nicht lästig fallen wird.

Mit ausgezeichneter Hochachtung

stets dankend

ergebenst F. Liszt

65. Friedrich Wilhelm Carl Bechstein, Gründer der Klavierfabrik C. Bechstein. Name des Adressaten und eine Signatur No. 107, von fremder Hand in deutscher Schrift geschrieben.

66. Pater Hermann Joseph Mohr, deutscher Kirchenmusik-Komponist, von Liszt sehr hoch geschätzt. Im Anhang teile ich hier einen unbekannten Liszt-Brief an Pater Mohr mit, der in der Manuskriptenabteilung der Bibliothèque Nationale in Paris aufbewahrt wird. 
15.

\title{
GSA 55/3934 \\ An einen deutschen Musikprofessor \\ [zwei beschriebene Seiten] ${ }^{67}$
}

Budapest, 8ten März [18]86

\section{Geehrter Herr Professor,}

Über Ihr beabsichtigtes „Wagner-Jahrbuch“ haben Sie sich mit Herrn von Wolzogen $^{68}$ zu verständigen. Die Bayreuther Blätter bieten seit Jahren ein fortgesetztes Wagner Buch; hierzu kommt noch das Wagner Lexicon von Glasenapp ${ }^{69}$ und Stein, ${ }^{70}$ die Wagner-Biographie // von Glasenapp, die Wagner Kalender ${ }^{71}$ und unzählige Schriftstücke... Man frägt sich was noch zu drucken wäre?

Meinerseits kann ich keine Beiträge liefern.

Freundlich dankend

F. Liszt

\author{
16. \\ GSA 59/71,12 \\ An Cécile Munkacsy \\ [leeres Couvert]
}

[ohne Datum]

\section{Frankreich}

Madame

Madame Cécile de Munkascy ${ }^{72}$

Avenue de Villiers 53

Paris

67. In der charakteristischen Schrift des schon schwer lesenden, augenkranken Liszt.

68. Hans von Wolzogen, Musikschriftsteller, Redakteur der Bayreuther Blätter.

69. Carl Friedrich Glasenapp, Dozent für die deutsche Sprache und Literatur in Riga, Verfasser von Richard Wagners Leben und Wirken, 2 Bde (Kassel - Basel, 1876-1877).

70. Heinrich von Stein, Philosoph und Schriftsteller, Mitherausgeber des Wagner-Lexikon: Hauptbegriffe der Kunst- und Weltanschauung Richard Wagners in wörtlichen Anführungen aus seinen Schriften (Stuttgart, 1883).

71. Emerich Kastner, Richard Wagner Kalender: Historische Daten aus des Meisters Leben und Wirken für die gesammte musikalische Welt (Wien: Fromme, 1883).

72. Frau des berühmten ungarischen Malers Mihály Munkácsy. Anlässlich seines letzten Pariser Aufenthaltes, im Frühling 1886, wohnte Liszt im prächtigen Palais der Munkácsys (s. obige Adresse). Unmittelbar vor seinem Tod besuchte Liszt das Ehepaar aus Bayreuth noch in ihrer Luxemburger Villa in Colpach. 


\section{Anhang \\ An Pater Hermann Joseph Mohr ${ }^{73}$ \\ [vier beschriebene Seiten]}

[mit Couvert, Poststempel Weimar 24.4.77]

Frankreich

Au très révérend Père

Joseph Mohr ${ }^{74}$

Rue des Fleurs 22

Toulouse

(France)

Weimar, 24 avril [18]77

Très révérend Père,

Je vous remercie respectueusement de l'envoi de votre Manuel de chant « contenant l'ordinaire de la Messe, les Psaumes et Hym[n]es etc. $\gg{ }^{75}$ Ce manuel est à la fois une oeuvre de piété et de profond et judicieux savoir musical.

En vous réglant, pour les trois premières parties, sur l'édition officielle de la sacrée congrégation des Rites, publiée par l'ordre et sous les auspices des Sa Sainteté Pie IX vous avez assuré à votre oeuvre son véritable poids d'authenticité, et son caractère d'universalité orthodoxe. La quatrième partie: «Cantiones Variae » mérite e[!]galement d'e[!]tre prise en sérieuse considération et activement propagée par les mai[!]tres de chapelle, les Regens chori, les organistes, les collaborateurs et les préposés à la musique d'église. On ne peut que louer le choix excellent des Hymnes dont vous avez au mieux assorti les tonalités, marqué les rhyt[h]mes sincères, et rendu l'exécution aisée par une notation correcte et précise.

Tant au point de vue de l'art religieux, qu'au point de vue du culte, il est fort désirable que votre Manuel devienne d'usage général dans les églises catholiques, et fructifie pieusement en des millions de voix et de coeurs, chantant la gloire et les bénédictions du Seigneur.

Veuillez agréer, très révérend Père, l'expression des mes sentiments de haute estime et respectueux dévouement.

F. Liszt

73. Paris, Bibliothèque Nationale, Département des Manuscrits, Rothschild A XIX 1685.

74. In der Musiksammlung der Bibliothèque Nationale in Paris befindet sich unter der Signatur MS 162 das Autograph von Liszts Anima Christi, sanctifica me [LW, S. 46/1, R. 495a], 1. Fassung: Für Männerchor und Orgel. 3 Seiten, rot überzogen: „Vide die 2te Version /4“ [autogr.], mit einer autographen Widmung am Schluss: „Au tres révérend Père Mohr S.J. son très respectueux et reconnaissant F.Liszt / 25 Juin 74 (Villa d'Este)“.

75. Manuel de chant : contenant l'ordinaire de la messe, les psaumes et hymnes des vêpres de toute l'année, et les complies, d'après l'édition de la Sacrée-Congrégation des Rites. Publiée par l'ordre et sous les auspices de Sa Sainteté Pie IX, suivi d'un recueil de cantiques latins et de prières pour les offices divines / par le P. Joseph Mohr, S.J. Avec l'approbation des supérieurs (Paris: Lethielleux, 1887). 
[Auf Seite 4, auf dem linken Rand, quer:]

Ci-joint le mot d'introduction que vous me demandez pour Gounod.

Etes-vous en relation avec l'abbé Gay vicaire de MS Pie, évèque de Poitiers ? Outre ses rares mérites et vertus ecclésiastiques, l'abbé Gay est aussi un musicien des plus compétens. Je vous engage à lui envoyer votre Manuel ; il saura l'apprécier plus dignement que moi, et à cette occasion redites lui ma vieille reconnaissance pour sa chrétienne bienveillance.

A quant l'édition à trois ou quatre voix de votre Manuel ?

Peut-être serait-il bon d'y ajouter un accompagnement d'orgue vu que la plupart des organistes ne sont guère au fait des harmonies que conviennent au chant grégorien et de ce qui s'ensuit. ${ }^{76}$

76. Liszt war um 1860 emsig um eine kirchenmusikalische Reform bemüht. In einem Brief an die Fürstin Carolyne vom 24. Juli 1860 schrieb er, hoffend, der Hl. Vater möge in einem Dekret den gregorianischen Gesang zum ausschließlichen Kirchengesang erklären. Alle Instrumente - ausgenommen die die Sänger unterstützende Orgel - würde er aus der Kirche verbannen. Von irgendeiner Harmonisierung der Choräle spricht er hier nicht. Er bittet die Fürstin, ihm den Rapporto Intorno a la Riforma della Musica die Chiesa, 1839, von Gasparo Spontini zu übersenden. Siehe Franz Liszt's Briefe, Bd. 5, Briefe an die Fürstin Carolyine Sayns-Wittgenstein, 2, herausgegeben von La Mara [Marie Lipsius] (Leipzig: Breitkopf und Härtel, 1900), 33-36. 


\section{7. \\ Mus.Schu 160 \\ [Albumblatt für Clara Schumann] $]^{77}$ \\ [ein Blatt] \\ [Liszts Musikhandschrift, drei Takte f. Klavier, A-Dur mit Unterschrift:]}

F. Liszt 27 März 40

FAKSIMILE 1 Albumblatt für Clara Schumann

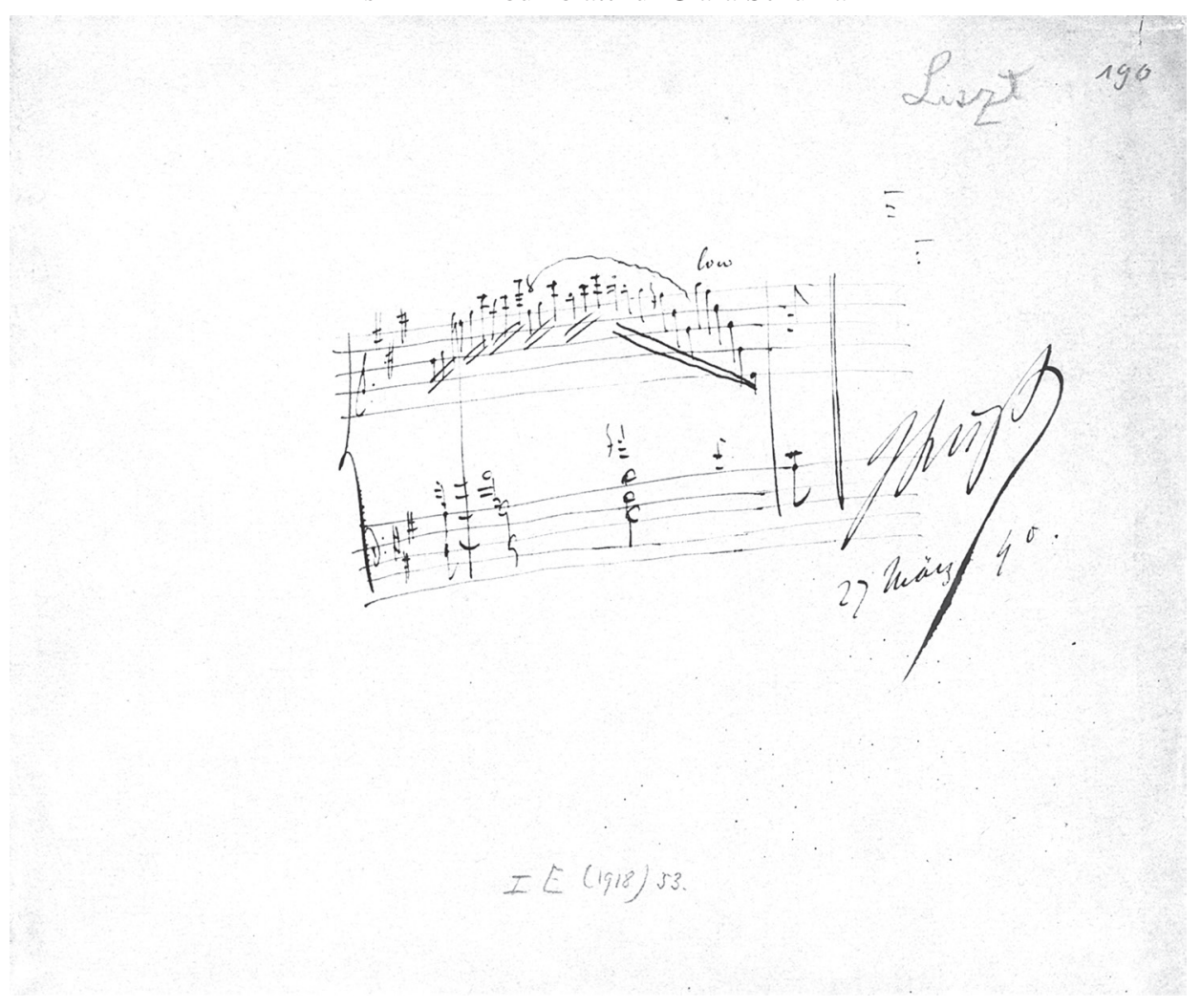

77. Liszt kam am 14. März 1840 in Dresden an und gab dort am 16. d. M. sein erstes Konzert. Schumann war von Leipzig herübergekommen, um ihn zu hören. Die Beiden lernten sich hier kennen, befreundeten sich und waren viel zusammen, reisten auch gemeinsam nach Leipzig (wo Liszt auch konzertierte). Am 27. und 29. März spielte Liszt wieder in Dresden. Zur Zeit hielt sich Clara Wieck in Berlin auf. In seinem am 18. März an sie geschriebenen Brief berichtet Robert seiner Geliebten ausführlich über seine ambivalenten Gefühle Liszt und seinem Klavierspiel gegenüber. Siehe Hanns-Josef Ortheil, Robert und Clara Schumann. Briefe einer Liebe (Königstein: Athenäum Verlag, 1982), 268-269. Die Identifizierung des Musikstückes ist mir leider nicht gelungen. Ich möchte mich ganz herzlich bei der Herausgeberin der Neuen Liszt-Ausgabe, Adrienne Kaczmarczyk bedanken, die sich freundlichst bemühte, mir dabei zu helfen. 
18.

\author{
Mus. Dresd. App. 722, 87 \\ [An eine unbekannte Gräfin] \\ [drei beschriebene Seiten]
}

Weymar, 4 février 1850

Madame La Contesse,

Le bienveillant souvenir que vous avez bien voulu garder de moi, et le gracieux envoi de vos Lieder que je viens de recevoir par l'intermédiaire de Mr le Baron de Hopfgarten ${ }^{78}$ me rendent deux fois obligé. Permettez moi de vous en exprimer mes remercimens et d'y joindre mes plus sincères complimens sur le sentiment distingué que respirent ces compositions vocales, d'ailleurs si bien // marqués au cachet des maîtres par la parfaite entente du tissu harmonique et la grâce des accompagnemens.

Mr d'Hopfgarten m'ayant fait espérer que nous aurions l'honneur de votre visite à Weymar dans le courant de cet hiver j'ajourne à ce moment ma réponse plus détaillée et motivée (laquelle je crains ne pourra être aussi favorable que je le desirerais) par rapport aux artistes que vous voulez bien me recommender.

Veuillez bien croire, Madame la Contesse que je ne me rendrai pas fautif d'un manque de zèle, ou de bien vouloir et daignez agréer, je vous prie, l'expression // des hommages les plus respectueux

de votre très humble serviteur

F. Liszt

\author{
19. \\ Mus. Dresd. App. 1375, 505 \\ [An Jean Louis Nicodé?] ${ }^{79}$ \\ [eine beschriebene Seite in deutscher Schrift]
}

Weimar 29 April 1884

Sehr geehrter Herr!

Entschuldigen Sie zunächst meine späte Beantwortung Ihres mir nach Paris gesandten Briefes.

78. Der Baron von Hopfgarten gehörte zu einer einflussreichen thüringischen Familie.

79. Der Adressat war vermutlich der Komponist und Dirigent Jean Louis Nicodé, dessen Symphonische Variationen Op. 271884 bei Breitkopf und Härtel erschienen, und 1886 tatsächlich auf das Programm der XXIII. Tonkünstler-Versammlung in Sondershausen gesetzt wurden. 
Ihre ganz vortefflichen symphonischen „Variationen“ [in lateinischer Schrift] kämen leider zu dem schon zu reichen Programm der diesjährigen Tonkünstlerversammlung am Weimar // $\mathrm{Tag}^{80}$ der übergroßen Fülle von Concerositismen, [welche] zu meinem Bedauern muß zur Aufführung gelangen und müßten wir uns diese Freude für die nächste Sammlung aufsparen.

Freundlichst ergebenst

F. Liszt

[Unten, in fremder Schrift, mit Bleistift:] Nur László Nemes ist eigenständig! ILN [??]

20.

Mscr. Dresd. App. 503 a-b

An Laura Kahrer ${ }^{81}$

[vierseitiges Briefpapier, eine beschriebene Seite]

[Mit Couvert: ${ }^{82}$

Deutschland

Fräulein

Laura Kahrer

Clavier Virtuosin

Lützowstrasse No 80, 2 Treppen

Berlin

Wien, 15ten April [18]69

Liebe erstaunliche Künstlerinn,

Empfangen Sie dies kleine Erinnerungszeichen an die Stunde wo mich ihr ausserordentliches Talent so freudig überraschte und seien Sie der aufrichtigen Wohlgewogenheit versichert mit welcher Ihnen verbleibt

F. Liszt

80. Die Festspiele der Tonkünstler-Versammlung in Weimar fanden zwischen dem 23. und 28. Mai statt. Liszt war Ehrenpräsident und stand das letzte Mal am Dirigentenpult. Von seinen Werken wurden aufgeführt: Die Legende von der Heiligen Elisabeth, Salve Polonia [aus dem Oratorium St. Stanislaus], die Graner Messe und Die Ideale.

81. Veröffentlicht in Franz Liszt's Briefe, Bd. 2, hrsg. von La Mara [Marie Lipsius] (Leipzig: Breitkopf und Härtel, 1893), 141. Als Anmerkung fügt die Herausgeberin hinzu: Der Brief wurde ,,von einer silbernen Feder zum Componiren“ begleitet, nachdem er ihrem ersten öffentlichen Auftreten im Wiener Hofoperntheater in einem Wohltätigkeitskonzert beigewohnt hatte.

82. Auf dem Poststempel: „Posta“, auf der Rückseite Krone mit Unicornis und Flügeln. Der Brief muss in Ungarn abgeschickt worden sein. 
21.

Mscr. Dresd. App. 503 c-d

An Laura Kahrer

[drei beschriebene Seiten]

[Mit Couvert:]

Fräulein

Laura Karer [sic]

FLiszt.

Sehr geehrtes Liebes Fräulein,

Pest, 13ten April [18]74

Obschon ich wenig Gelegenheit hatte Ihnen mein aufrichtig wohlwollendes Interesse zu bezeigen, verbleibt es ungeschmälert.

Gewisse musikalische Mißverhältnisse sind mir zu bekannt: deshalb verzichte ich auf jedwede Einmischung - und liess Ihr vorletztes Schreiben unbeantwortet. Auch heute bin ich etwas verlegen, bezüglich der von Ihnen gewünschten Empfehlung. Ob Baron Hans von Bülow, im Laufe des Sommers in Deutschland // verweilt, ist mir noch unbekannt und wenn er sich dazu entschliesst wird er gewiss sehr der Ruhe bedürfen, - folglich wenig disponirt sein selbst liebenswürdige und ausgezeichnete Pianisten zu hören.

Indessen, wollen Sie liebes Fräulein ihrem Glück vertrauen und Bülow aufsuchen so zeigen Sie Ihm diese Zeilen ${ }^{83}$ womit Sie wiederholt versichert, dass in angenehmster Erinnerung ihres Talents und ihrer Persönlichkeit Ihnen stets verbleibt

freundlichst gesinnt

F. Liszt

[Ganz unten und auf S. 3 unten fortgesetzt:]

An Professor Weitzmann ${ }^{84}$ bitte ich meinen // hochachtungsvollen und freundschaftlichen Gruss zu sagen.

e) Zeichnung [Schloßgartentor?] 
22.

Mscr. Dresd. App. 1551, 190

An Monsieur Lindemann

[zwei beschriebene Seiten]

[Mit Couvert mit rotem Wachsstempel:]

FLiszt -

Weymar 28 Août [nach 1851]

Un artiste de beaucoup d'esprit et de talent, Monsieur Ehrlich de Vienne, devant passer quelques jours à Hanovre je crois vous être agréable, mon cher Monsieur, en vous le présentant et le recommandant à votre obligeance par ces lignes. Il vous suffira de l'entendre quelques minutes pour apprécier à quel point est justifié l'intérêt que je lui porte, et si les conjonctures lui etaient favorables à Hanovre, je suis persuadé que son talent de Pianiste y trouverait un parfait succès.

Veuillez bien agréer, je vous // prie, mon cher Monsieur, l'expression des sentimens les plus distingués et les plus affectueux

de votre compagnon

d'infortune d'Eilsen $^{85}$

FLiszt

85. Liszts Lebensgefährtin, die Fürstin Carolyne von Sayn-Wittgenstein, und ihre Tochter Marie weilten öfter in Bad Eilsen zur Chur. Liszt selbst besuchte sie dort öfter und weilte 1849, 1851 und 1851 insgesamt, verteilt, neun Monate bei ihnen. Siehe Klaus Leimenstoll, „,Franz Liszt in Eilsen in den Jahren 1849, 1850 und 1851“ (Manuskript, Eilsen, 2008). Liszt veranstaltete dort am 3. Juli 1851 ein Konzert für die junge, begabte Harfenkünstlerin Rosalie Spohr, die spätere Gräfin Sauerma, Nichte des Komponisten Louis Spohr. Als Mitwirkenden engagierte er Herrn Lindemann, „Un amateur distingué ... de Hannovre [qui] m’a promis de jouer un ou deux Solos sur le Violoncelle et le reste du programme se donnera aisément“. Siehe Franz Liszt's Briefe, Bd. 1, 100-101. Was für ein „Missgeschick“ bei diesem Konzert geschehen mochte, ist nicht nachweisbar. 
23.

Mscr. Dresd. Aut. 154 a-f

An Bertrand Roth

[eine beschriebene Seite]

[Mit Couvert:]

Deutschland

Herrn

Bertrand Roth

Stud.: Mus: philoso

Neumarkt

42, IV

Leipzig

Geehrter Herr,

Budapest, 1sten März [18]77

Freundlichst dankend für Ihre Zeilen, versichert Sie des besten Empfanges in

Weimar, wo Anfangs April eintreffen wird

F. Liszt

24.

Mscr. Dresd. Aut. 1400, 5a

An Bertrand Roth

[Visitenkarte]

[Mit Couvert:]

Herrn

Bertrand Roth + [unleserlich]

Plauen

Starnbergstrasse

Zum Bienengarten

[Poststempel: 5479 Weimar]

F. Liszt. [Handschrift]: freundlichst erwartet sie 
25.

Mus. Dresd. Aut. 1400, 5b

An J. J. Fr. Roth ${ }^{86}$

[zwei beschriebene, lose Blätter]

[Couvert mit ungarischer Briefmarke Krone 5, Magyar Kir. Posta:]

Deutschland

Herrn J. J. Fr. Roth.

zum Bienengarten

$\underline{\text { Plauen }}$

(Sachsen)

Budapest, 29ten Jänner, [18]78

Sehr geehrter Herr,

Mit aufrichtigem Vergnügen antworte ich:

Ihr Herr Sohn, Bertrand Roth, ist ein vortrefflicher, gediegener und der besten Erfolge werther Künstler. Als solcher, wird er überall Anerkennung und Geltung finden, trotz mancherlei Schwierigkeiten welche sich in der musikalischen Wirthschaft einstellen.

Sein Talent ist sehr // ausgebildet, und schreitet rüstig vorwärts.

Nun soll er Ihnen, geehrter Herr, immer mehr Freude und Ehre bringen.

ergebenst

F.Liszt 
26.

Mscr. Dresd. Aut. 1546b

An Bertrand Roth

[Visitenkarte]

[Mit Couvert, ohne Ort und Datum (nicht per Post):]

Herrn Roth

FL

[Auf der Rückseite der Karte:]

[Ohne Ort und Datum]

Lieber Freund,

Besitzen Sie nicht die Härtel'sche Ausgabe Beethoven's Concerte mit dem arrangement für ein zweites Clavier von Moscheles?

Wenn ja, schicken Sie mir dieselbe sogleich.

Heute, halb 4 Uhr erwartet Sie freundlichst

FL

27.

Mscr. Dresd. Aut. 546c

An Bertrand Roth

[vier Seiten, zwei beschriebene Blätter]

[Mit Couvert (nicht per Post):]

Herrn

Herrn Bertrand Roth.

Via dei Cappuccini 6.

[Roma]

Lieber Roth,

Villa d'Este. 28 Nov: [18]78.

Gehen sie morgen, zwischen 1-2 Uhr zu Excellencz von Keudell $;{ }^{87}$ presentiren sie Ihnen ihr Conzert Programm; und besprechen sie mit Sgambati ${ }^{88}$ (der die Freundlichkit hat Ihnen // diese Zeilen zu überbringen), an welchen Tag ihr römisches Concert, am besten statt finden kann.

Freundichst ergebenst

F. Liszt

87. Robert von Keudell, langjähriger, musikliebender Gesandte in Rom.

88. Giovanni Sgambati, Pianist und Komponist, Liszts italienischer Schüler. 


\section{8.}

Mscr. Dresd. Aut. 1546d

An Bertrand Roth

[eine Seite]

[Mit Couvert, nicht per Post:]

Herrn

Herrn Bertrand Roth.

Via dei Cappuccini

No 6

[Roma]

Villa d'Este, Freitag (6 December)

Das Programm ihres römischen Conzerts ${ }^{89}$ nimmt sich gedruckt vortrefflich aus: noch schöner wird sich das Zuhören und applaudiren machen. Auf Wiedersehen nächsten Donnerstag, Lieber Roth. Freundschaftlich ergebenst

FLiszt

29.

Mscr. Dresd. Aut. 1546e

An Bertrand Roth

[vier Blätter, eine beschriebene Seite]

[Mit Couvert Eingeschrieben:]

Deutschland

Herrn Bertrand Roth

zum Bienengarten

Plauen

(Voigtland)

$\underline{\text { Sachsen }}$

[Auf der Rückseite: 4 ungarische Briefmarken mit Krone und Stempel. Budapest 26 Januar 1880] 
Geehrter Freund,

Budapest, 26ten Januar [18]80

Nur sehr ausnahmsweise schreibe ich Empfehlungszeilen: insbesondere an Ihre Excellenz Frau Gräfin von Schleinitz ${ }^{90}$ und Herrn Bechstein. ${ }^{91}$

Beiliegend die zwei verlangten Briefchen, denen die herzlichsten Glückwünsche zu ihrer Vermählung beifügt,

freundschaftlich ergebenst

F. Liszt

FAKSIMILE 2 Brief 29
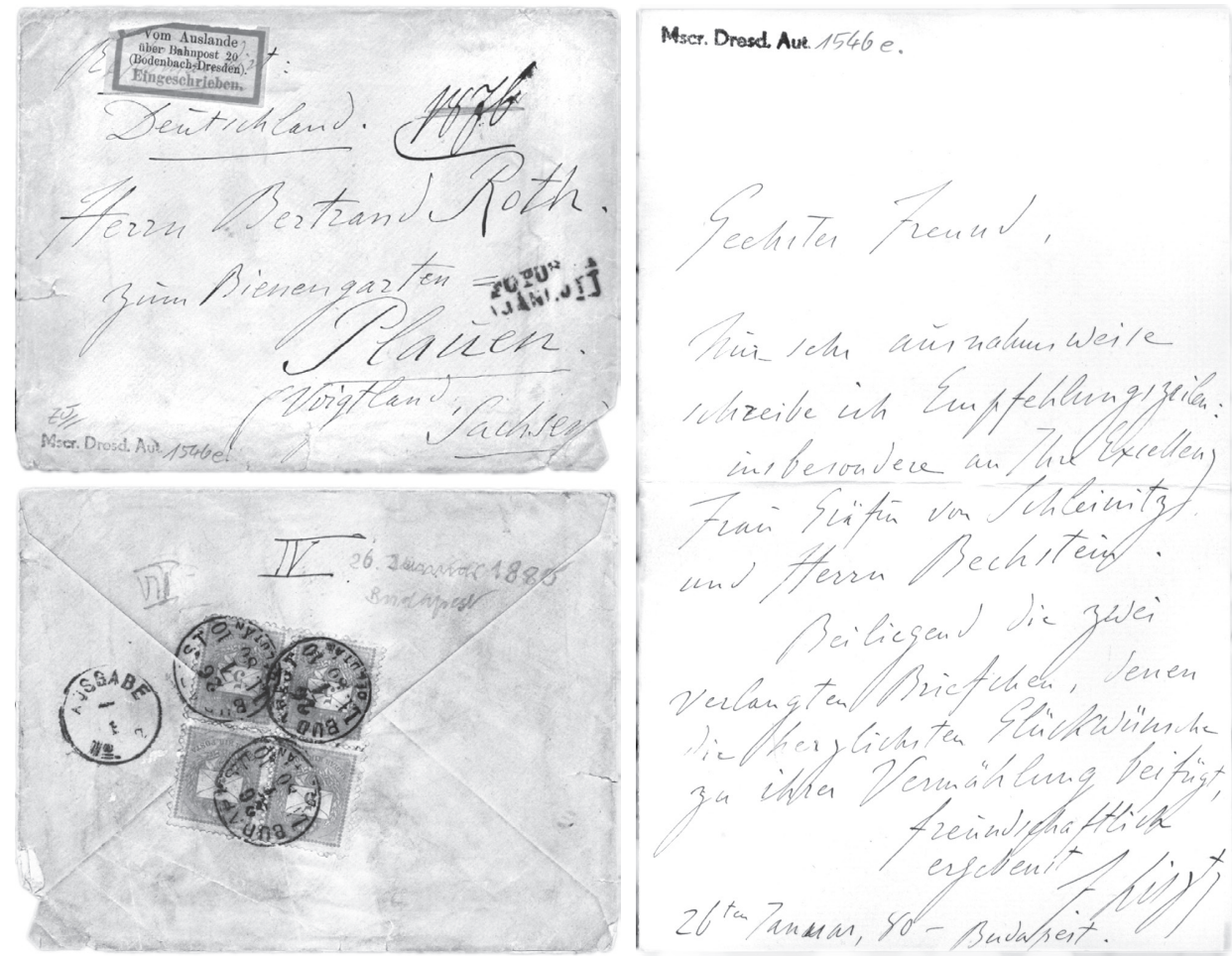

90. Marie von Schleinitz, geb. von Buch, Gattin des preußischen Ministers Alexander von Schleinitz, später mit Anton Graf von Wolkenstein verheiratet. Gönnerin Liszts und Wagners, intime Freundin Cosimas.

91. Carl Friedrich Wilhelm Bechstein, deutscher Klavierfabrikant. 
30 .

Mscr. Dresd. Aut. 1546f

An Bertrand Roth

[ein beschriebenes Blatt]

[Mit Couvert, Poststempel Weimar:]

Herrn

Bertrand Roth

Plauen

(Sachs. Voigtland)

zum Bienengarten

Weimar, 21sten April [18]80 -

Bestens willkommen in Weimar, geehrter Freund. Ausgenommen die Woche vom 18ten bis 24 Mai, wo ich der „Tonkünstler Versammlung“ in Baden-Baden obliege, treffen Sie hier,

aufrichtig ergebenst

F. Liszt 